\title{
TUNNELING WITH DISSIPATION IN OPEN QUANTUM SYSTEMS
}

\author{
G.G.Adamian ${ }^{1,2}$, N.V.Antonenko ${ }^{1,2}$ and W.Scheid ${ }^{1}$ \\ ${ }^{1}$ Institut für Theoretische Physik der Justus-Liebig-Universität, D-35392 Giessen, Germany \\ ${ }^{2}$ Joint Institute for Nuclear Research, 141980 Dubna, Russia
}

(August 12, 2018)

\begin{abstract}
Based on the general form of the master equation for open quantum systems the tunneling is considered. Using the path integral technique a simple closed form expression for the tunneling rate through a parabolic barrier is obtained. The tunneling in the open quantum systems strongly depends on the coupling with environment. We found the cases when the dissipation prohibits tunneling through the barrier but decreases the crossing of the barrier for the energies above the barrier. As a particular application, the case of decay from the metastable state is considered.
\end{abstract}

PACS: 03.65.-w, 05.30.-d, 24.60.-k 
There has been considerable interest to the quantum tunneling of a particle through an energy barrier when the dissipation is present 15 . Using various models for the description of the quantum open system, the opposite dependences of tunneling rate on the dissipation have been observed. It is generally thought that tunneling probability decreases in the presence of coupling to the environment. Disregarding the stage of averaging over the intrinsic degrees of freedom, one can consider the tunneling effect starting right away from the general Markovian master equation for the reduced density matrix of the collective degree of freedom $16-25$

$$
\frac{d \hat{\rho}(t)}{d t}=-\frac{i}{\hbar}\left[\hat{H}_{0}, \hat{\rho}\right]+\frac{1}{2 \hbar} \sum_{j}\left(\left[\hat{V}_{j} \hat{\rho}, \hat{V}_{j}^{+}\right]+\left[\hat{V}_{j}, \hat{\rho} \hat{V}_{j}^{+}\right]\right) .
$$

Here, $\hat{H}_{0}$ is the Hamiltonian of the collective subsystem and $\hat{V}_{j}$ are operators acting in the Hilbert space of the subsystem. The second term in (11) is responsible for the friction and diffusion and supplies the unreversability in the open quantum system. Omitting this term we get a standard form for the density matrix evolution equation in the case of closed system. The generality of Eq. (何) was mathematically proved in 18 , 19 .

In the one-dimensional case the phase space path integral expression 26 for the propagator corresponding to (1) is written as

$$
\begin{aligned}
G\left(q, q^{\prime}, t ; q_{0}, q_{0}^{\prime}, 0\right) & =\int_{q_{0}(0)}^{q(t)} D[\alpha] \int_{q_{0}^{\prime}(0)}^{q^{\prime}(t)} D\left[\alpha^{\prime}\right] \exp \left(\frac{i}{\hbar} S\left[\alpha, \alpha^{\prime}\right]\right), \\
S\left[\alpha, \alpha^{\prime}\right] & =\int_{0}^{t} d \tau\left\{\dot{q}(\tau) p(\tau)-H_{e f f}(q(\tau), p(\tau))\right\} \\
& -\int_{0}^{t} d \tau\left\{\dot{q}^{\prime}(\tau) p^{\prime}(\tau)-H_{e f f}^{*}\left(q^{\prime}(\tau), p^{\prime}(\tau)\right)\right\} \\
& -i \sum_{j} \int_{0}^{t} d \tau\left\{V_{j}(q(\tau), p(\tau)) V_{j}^{*}\left(q^{\prime}(\tau), p^{\prime}(\tau)\right)\right\},
\end{aligned}
$$

with the effective Hamiltonian

$$
H_{e f f}=H_{0}-\frac{i}{2} \sum_{j}\left|V_{j}\right|^{2}
$$


Here, the Wigner transform of operators $\hat{H}_{0} \hat{V}_{j}^{+} \hat{V}_{j}, \hat{V}_{j}^{+}$and $\hat{V}_{j}$ are denoted by $H_{0},\left|V_{j}\right|^{2}, V_{j}^{*}$ and $V_{j}$, respectively. For the inverted harmonic oscillator with the Hamiltonian

$$
\hat{H}_{0}=\frac{1}{2 m} \hat{p}^{2}-\frac{m \omega^{2}}{2} \hat{q}^{2}
$$

and linear environment operators

$$
\hat{V}_{j}=A_{j} \hat{p}+B_{j} \hat{q}, \quad \hat{V}_{j}^{+}=A_{j}^{*} \hat{p}+B_{j}^{*} \hat{q}, \quad j=1,2,
$$

the propagator (2) can be evaluated analytically:

$$
G\left(q, q^{\prime}, t ; q_{0}, q_{0}^{\prime}, 0\right)=\frac{m \omega}{2 \pi \hbar \sinh (\omega t)} \exp (\lambda t) \exp \left(i S_{R} / \hbar\right) \exp \left(-S_{I} / \hbar\right)
$$

where

$$
\begin{aligned}
& S_{R}=\frac{m \omega}{2 \sinh (\omega t)}\left(\cosh (\omega t)\left[q_{0}^{2}-\left(q_{0}^{\prime}\right)^{2}+q^{2}-\left(q^{\prime}\right)^{2}\right]\right. \\
&-\left.2 \cosh (\lambda t)\left[q_{0} q-q_{0}^{\prime} q^{\prime}\right]-2 \sinh (\lambda t)\left[q_{0} q^{\prime}-q_{0}^{\prime} q\right]\right), \\
& S_{I}=\frac{m}{8 \lambda\left(\omega^{2}-\lambda^{2}\right) \sinh ^{2}(\omega t)}\left(\mathcal{A}(t)\left[q_{0}-q_{0}^{\prime}\right]^{2}-4 \mathcal{B}(t)\left[q_{0}-q_{0}^{\prime}\right]\left[q-q^{\prime}\right]-\mathcal{A}(-t)\left[q-q^{\prime}\right]^{2}\right), \\
& \mathcal{A}(t)=a \exp (2 \lambda t)+b \sinh (2 \omega t)+c \cosh (2 \omega t)-d, \\
& \mathcal{B}(t)=a \cosh (\omega t) \sinh (\lambda t)+b \sinh (\omega t) \cosh (\lambda t), \\
& a=\frac{2 \omega^{2}}{\hbar m}\left(m^{2} D_{q q}\left[\omega^{2}-2 \lambda^{2}\right]-2 \lambda m D_{p q}-D_{p p}\right), \\
& b=\frac{2 \omega \lambda}{\hbar m}\left(m^{2} \omega^{2} D_{q q}+2 \lambda m D_{p q}+D_{p p}\right), \\
& c=\frac{2 \lambda}{\hbar m}\left(m^{2} \omega^{2} \lambda D_{q q}+\lambda D_{p p}+2 m \omega^{2} D_{p q}\right), \\
& d=\frac{2}{\hbar m}\left(\omega^{2}-\lambda^{2}\right)\left(m^{2} \omega^{2} D_{q q}-D_{p p}\right) .
\end{aligned}
$$

Here, the quantum mechanical diffusion coefficients $D_{q q}=\frac{\hbar}{2} \sum_{j}\left|A_{j}\right|^{2}, D_{p p}=\frac{\hbar}{2} \sum_{j}\left|B_{j}\right|^{2}$ and $D_{q p}=-\frac{\hbar}{2} \operatorname{Re} \sum_{j} A_{j}^{*} B_{j}$ and the frictional damping rate $\lambda=-\operatorname{Im} \sum_{j} A_{j}^{*} B_{j} 18.29225$ satisfy the following constraints: $D_{q q}>0, D_{p p}>0$ and $D_{p p} D_{q q}-D_{p q}^{2} \geq \lambda^{2} \hbar^{2} / 4$ which secure the non-negativity of the density matrix at any time. The diffusion models, in which these constraints are not fulfilled, can be related to the classical or semiclassical considerations because they allow the violation of the uncertainty inequality at some time $15,20,24$. 
Using (5), $\hat{\rho}(t)$ is determined from $\hat{\rho}(t=0)$ as

$$
<q|\hat{\rho}(t)| q^{\prime}>=\int d q_{0} \int d q_{0}^{\prime} G\left(q, q^{\prime}, t ; q_{0}, q_{0}^{\prime}, 0\right)<q_{0}|\hat{\rho}(t=0)| q_{0}^{\prime}>
$$

In order to study the tunneling with the Hamiltonian (3), we consider a particle in the initial state

$$
\Psi(q)=\left(2 \pi \sigma_{q q}(0)\right)^{-1 / 4} \exp \left(-\frac{1}{4 \sigma_{q q}(0)}(q-\bar{q}(0))^{2}+\frac{i}{\hbar} \bar{p}(0) q\right)
$$

in the left-hand side from the potential barrier. The calculation of (6) with (5) and (7) yields the Gaussian distribution at time $t$

$$
\rho(q, t)=<q|\hat{\rho}(t)| q>=\left(2 \pi \sigma_{q q}(t)\right)^{-1 / 2} \exp \left(-\frac{1}{2 \sigma_{q q}(t)}(q-\bar{q}(t))^{2}\right)
$$

with the first $\bar{q}(t)$ and second $\sigma_{q q}(t)$ moments. The equations for these moments are given in Refs.15, 18, 19,21 24 and below for arbitrary potential. Originally they contain the friction in both coordinate $\lambda_{q}$ and momentum $\lambda_{p}$ so that $\lambda_{p}+\lambda_{q}=2 \lambda$. The considered particular case of $\lambda_{p}=\lambda_{q}=\lambda$ is generalized for $\lambda_{p} \neq \lambda_{q}$ by using the canonical transformations 15 $p^{\prime}=p+\mu m q, q^{\prime}=q$ with the parameter $\mu$. Therefore, the expression (8) can be applied to the case of $\lambda_{p} \neq \lambda_{q}$ as well.

The solutions of equations for the first and second moments in (8) are

$$
\begin{aligned}
\bar{q}(t) & =e^{-\lambda t}\left(\bar{q}(0)\left[\cosh (\psi t / 2)+\frac{\lambda_{p}-\lambda_{q}}{\psi} \sinh (\psi t / 2)\right]+\frac{2}{m \psi} \bar{p}(0) \sinh (\psi t / 2)\right), \\
\sigma_{q q}(t) & =\frac{1}{2 m^{2} \lambda\left(\omega^{2}-\lambda_{p} \lambda_{q}\right)}\left[m^{2}\left(\omega^{2}-2 \lambda_{p} \lambda\right) D_{q q}-D_{p p}-2 m \lambda_{p} D_{p q}\right] \\
& +e^{-2 \lambda t}\left[\frac{2 C_{1}}{m\left(\lambda_{q}-\lambda_{p}\right)}-\frac{1}{2 m \omega^{2}}\left[\left(\lambda_{q}-\lambda_{p}\right) C_{2}+C_{3} \psi\right] \cosh (\psi t)\right. \\
& \left.+\frac{1}{2 m \omega^{2}}\left[\left(\lambda_{q}-\lambda_{p}\right) C_{3}+C_{2} \psi\right] \sinh (\psi t)\right],
\end{aligned}
$$

where the following notations are used:

$$
\begin{aligned}
C_{1} & =\frac{m \omega^{2}\left(\lambda_{q}-\lambda_{p}\right)}{\psi^{2}}\left[\sigma_{q q}(0)-\frac{1}{m^{2} \omega^{2}} \sigma_{p p}(0)+\frac{\lambda_{q}-\lambda_{p}}{m \omega^{2}} \sigma_{p q}(0)\right. \\
& \left.-\frac{1}{\lambda} D_{q q}+\frac{1}{m^{2} \omega^{2} \lambda} D_{p p}-\frac{\left(\lambda_{q}-\lambda_{p}\right)}{m \omega^{2} \lambda} D_{p q}\right]
\end{aligned}
$$




$$
\begin{aligned}
C_{2} & =\frac{1}{\psi^{2}}\left[\frac{\lambda_{q}-\lambda_{p}}{m}\left(\sigma_{p p}(0)-m^{2} \omega^{2} \sigma_{q q}(0)\right)+4 \omega^{2} \sigma_{p q}(0)\right. \\
& \left.+\frac{1}{\omega^{2}-\lambda_{p} \lambda_{q}}\left(\frac{2 \omega^{2}-\lambda_{p} \lambda_{q}+\lambda_{q}^{2}}{m}\left[D_{p p}+m^{2} \omega^{2} D_{q q}\right]+4 \lambda \omega^{2} D_{p q}\right)\right], \\
C_{3} & =-\frac{1}{m \psi}\left[m^{2} \omega^{2} \sigma_{q q}(0)+\sigma_{p p}(0)\right. \\
& \left.+\frac{1}{\omega^{2}-\lambda_{p} \lambda_{q}}\left(\lambda_{q} D_{p p}+2 m \omega^{2} D_{p q}+m^{2} \omega^{2} \lambda_{p} D_{q q}\right)\right]
\end{aligned}
$$

and $\psi=\sqrt{\left(\lambda_{p}-\lambda_{q}\right)^{2}+4 \omega^{2}}$. With these expressions we obtain the same result as in Ref.27 at $\lambda_{p}=\lambda_{q}=0, D_{p p}=D_{q q}=D_{p q}=0$ and $\sigma_{p p}(0)=\hbar^{2} /\left(4 \sigma_{q q}(0)\right)\left(\sigma_{q p}(0)=0\right)$. For $\lambda_{q}=0$, $D_{p p}=D_{q q}=D_{p q}=0$ and $\sigma_{p p}(0)=\hbar^{2} /\left(4 \sigma_{q q}(0)\right)\left(\sigma_{q p}(0)=0\right)$, our results coincide in the underdamped limit with the results of Ref. inverted Caldirola-Kanai Hamiltonian.

The penetration probability at time $t$ is determined by the following expression $(q=0$ corresponds to the top of the barrier):

$$
P(t)=\int_{0}^{\infty} d q[\rho(q, t)-\rho(q, t=0)] / \int_{-\infty}^{0} d q \rho(q, 0),
$$

which is the ratio of change of the probability to be on the right-hand side of the barrier in time $t$ over the initial probability of the finding the particle on the entry left-hand side. Using Eqs. (8)-(10), the penetration probability $P=P(t \rightarrow \infty)$ is easially calculated taking the initial variances in accordance with the uncertainty relation. Here, we use $\sigma_{q q}(0) \sigma_{p p}(0)=$ $\hbar^{2} / 4$ and $\sigma_{p q}(0)=0$.

The dependences of the penetration probability through the parabolic barrier on the initial energy $E$ of system are presented in Fig. 1 for three sets of the friction coefficients $\lambda_{p}$ and $\lambda_{q}$. All diffusion coefficients depend only on $\lambda$. For the sub-barrier energies $(E<$ $0)$, the tunneling is larger for $\lambda_{q}=\lambda_{p} \neq 0$ as comparable to the case without friction $\lambda_{p}=\lambda_{q}=0$. For $E<0$, the dissipation in coordinate $\lambda_{q}$ increases but dissipation in momentum $\lambda_{p}$ decreases the barrier penetration. The increase of the tunneling was obtained in the microscopic Gisin's model2 for large friction. However, in this model one can not distinguish the influence of frictions in coordinate and momentum on the tunneling. Larger 
penetration of the barrier than in the standard coupled-channel calculations is necessary to explain the experimental data on the sub-barrier fusion 29. It could be that in this case the coupling with environment leads to $\lambda_{q} \neq 0$ that renormalizes the barrier and increases the penetration 5 . The friction and diffusion reduce the crossing of the barrier for the energies above the barrier. For $E=0$ and $\lambda_{p}=\lambda_{q}$, the penetration and reflection probabilities are equal to each other with and without dissipation.

In Fig. 2 we show how the tunneling depends on the diffusion coefficients at different values of friction in momentum $\lambda_{p}$ for $\lambda_{q}=0$. One can see that only with the diffusion coefficient in momentum $D_{p p}\left(D_{q q}=D_{p q}=0\right) P$ decreases with increasing $\lambda_{p}$. Note that this set of diffusion coefficients is not compatible with the quantum mechanical consideration. For $D_{q q} \neq 0$, the value of $P$ initially decreases with increasing $\lambda_{p}$ up to some "critical" friction coefficients and then it starts to grow. This effect becomes more evident at larger $D_{q q}$ and $D_{p p}$ (higher temperature). The "critical" friction coefficient decreases with increasing temperature. This behaviour of the tunneling probability $P$ as a function of $\lambda_{p}$ can be explained in the following way: The tunneling is more crucial to the value of $D_{q q}$ then to the value of $D_{p p}$ because $\sigma_{q q}(t)$ (correspondingly $\rho(q, t)$ and $P$ ) is more sensitive to $D_{q q}$ than to $D_{p p}$; At large $\lambda_{p}$ the system has a longer time for the tunneling and during this time $\sigma_{q q}(t)$ and $P(t)$ strongly increase due to diffusion in coordinate. The increase of tunneling rate with temperature is in agreement with Ref目.

The probability of finding the particle to the right of the barrier is very sensitive to the width $\sigma_{q q}(0)$ of the initial wave packet localized to the left of the barrier at $t=0$ (Fig. 3). This effect is weaker with the dissipation. For smaller $\sigma_{q q}(0)$, the value of $\sigma_{p p}(0)$ becomes larger in quantum mechanics and the penetration probability increases due to the larger fluctuation energy. In the vicinity of $\sigma_{q q}(0)=\hbar /(2 m \omega)$ the dependence of $P$ on $\sigma_{q q}(0)$ becomes weak and the curve in fig.3 has a step-like behaviour.

The calculated time of decay from the metastable state in the potential

$$
U(q)=\alpha q^{2}-\beta q^{3}
$$


is shown in Fig. 4 as a function of $\lambda_{p}$. These data result from the solution of equations for the first and second moments (obtained from eq. (11)):

$$
\begin{aligned}
\frac{d \bar{q}}{d t} & =-\lambda_{q} \bar{q}+\frac{1}{m} \bar{p} \\
\frac{d \bar{p}}{d t} & =-\partial U(\bar{q}) / \partial \bar{q}-\frac{1}{2} \partial^{3} U(\bar{q}) / \partial \bar{q}^{3} \sigma_{q q}-\lambda_{p} \bar{p} \\
\frac{d \sigma_{q q}}{d t} & =-2 \lambda_{q} \sigma_{q q}+\frac{2}{m} \sigma_{p q}+2 D_{q q} \\
\frac{d \sigma_{p p}}{d t} & =-2 \lambda_{p} \sigma_{p p}-2 \partial^{2} U(\bar{q}) / \partial \bar{q}^{2} \sigma_{p q}+2 D_{p p} \\
\frac{d \sigma_{p q}}{d t} & =-\partial^{2} U(\bar{q}) / \partial \bar{q}^{2} \sigma_{q q}+\frac{1}{m} \sigma_{p p}-\left(\lambda_{p}+\lambda_{q}\right) \sigma_{p q}+2 D_{p q}
\end{aligned}
$$

These equations are obtained from (1) and (4) for arbitrary potential $U(q)$. In order to calculate $P(t)$ for short times, we can use in the first approximation the formalism elaborated for the parabolic barrier. The value of time $t_{1 / 2}$ at which $P\left(t_{1 / 2}\right)=0.5$ (the value of $\bar{q}$ corresponds to the top of the barrier) may be defined in some sense as the tunneling timet. The tunneling time increases monotonically with $\lambda_{p}\left(\lambda_{q}=0\right)$ when $D_{q q}=0$. For $D_{q q} \neq 0$, the value of $t_{1 / 2}$ initially increases with $\lambda_{p}$ and then it decreases. This means that for large $\lambda_{p}$ the dissipation prohibits the decay from metastable state due to diffusion in coordinate. The results of calculations above are in agreement with the results obtained int using the Gisin modele8 for the double well potential.

In conclusion, our calculations show that the dissipative effects on the tunneling process are quite complicated. It is evident that the earlier conclusions that the dissipation inhibit tunneling is not correct in the general case. There are examples when the dissipation prohibits the penetration through the barrier. Using the general master equation (1) for describing the open quantum systems, we can transparently show the influence of each friction and diffusion coefficient on the tunneling. However, the microscopical calculation of these coefficients in the real system remains to be interesting problem. In the consistent quantum treatment the tunneling should be calculated with the set of the diffusion coefficients where $D_{q q}>0$. As was shown, the tunneling is crucial to the value of $D_{q q}>0$. If the environment operators lead to $\lambda_{q} \neq 0$ then the interaction with environment renormalizes 
the potential barrier and influence the tunneling. With the initial Gaussian distribution (7) the distribution function remains to be Gaussian at any time.

The author (N.V.A.) is grateful to the Alexander von Humboldt-Stiftung for the financial support. This work was supported in part by DFG. 


\section{REFERENCES}

${ }^{1}$ Harris E.G. 1993 Phys. Rev. A 48995.

${ }^{2}$ Fujikawa K., Iso S., Sasaki M., and Suzuki H. 1992 Phys. Rev. Lett. 681093.

${ }^{3}$ Weiss U 1992 Quantum dissipative systems (Singapore: World Scientific)

${ }^{4}$ Baskoutas S. and Jannussis A. 1992 J. Phys. A: Math. Gen. 25 L1299.

${ }^{5}$ Ford G.W., Lewis J.T., and O'Connel R.F. 1991 Phys. Lett. A 158367.

${ }^{6}$ Razavy M 1990 Phys. Rev. A 416668.

${ }^{7}$ Razavy M, Pimpale A 1988 Phys. Rep. 168305

${ }^{8}$ Caldeira A.O. and Leggett A.J. 1981 Phys. Rev. Lett. 46211.

${ }^{9}$ Caldeira A.O. and Leggett A.J. 1983 Ann. Phys. 149374.

${ }^{10}$ Widom A. and Clark T.D. 1982 Phys. Rev. Lett. 4863.

${ }^{11}$ Widom A. and Clark T.D. 1982 Phys. Rev. Lett. 481571.

12 Bruinsma R. and Per Bak 1986 Phys. Rev. Lett. 56420.

${ }^{13}$ Widom A. and Clark T.D. 1984 Phys. Rev. B 301205.

${ }^{14}$ Leggett A.J. 1984 Phys. Rev. B 301208.

${ }^{15}$ Adamian G G, Antonenko N V, Scheid W 1997 J. Phys. A: Math. Gen. submitted.

${ }^{16}$ Belavin A A, Zel'dovich B Ya, Perelomov A M, Popov B S 1969 JTEP 56264

${ }^{17}$ Davies E B 1976 Quantum theory of open systems (New York: Academic Press)

${ }^{18}$ Lindblad G 1976 Commun. Math. Phys. 48119

${ }^{19}$ Lindblad G 1976 Rep. on Math. Phys. 10393

${ }^{20}$ Dekker H 1981 Phys. Rep. 801 
${ }^{21}$ Dodonov V V, Man'ko 1986 Reports of Physical Institute 1677

${ }^{22}$ Sandulescu A and Scutaru H 1987 Ann. Phys. (N.Y.) 173277

${ }^{23}$ Isar A, Sandulescu A, Scutaru H, Stefanescu, Scheid W 1994 Intern. J. Mod. Phys. A 3 635

${ }^{24}$ Isar A, Sandulescu A and Scheid W 1993 J. Math. Phys. 343887

${ }^{25}$ Antonenko N V, Ivanova S P, Jolos R V, Scheid W 1994 J. Phys. G: Nucl.Part.Phys. 20 1447

${ }^{26}$ Strunz W.T. 1997 J. Phys. A: Math. Gen. 304053.

${ }^{27}$ Papadopoulos G.J. 1990 J. Phys. A: Math. Gen. 23935.

${ }^{28}$ Gisin 1980 Physica A 111364

${ }^{29}$ K.Hagino et al. Phys. Rev. Lett. 79 (1997) 2014. 


\section{FIGURES}

FIG. 1. Calculated dependence of the penetration probability through the parabolic barrier on the initial energy of particle $E$ at temperature $k T=0, \hbar \omega=2.0 \mathrm{MeV}, q(0)=-2 \mathrm{fm}, \sigma_{q q}(0)=0.2$ $\mathrm{fm}^{2}, m=53 m_{0}$ ( $m_{0}$ is the mass of nucleon), $D_{q q}=\hbar \lambda /(2 m \omega), D_{p p}=\lambda m \hbar \omega / 2$ and $D_{p q}=0$. The results for the cases $\left(\lambda_{p}=\lambda_{q}=0\right),\left(\hbar \lambda_{p}=\hbar \lambda_{q}=1 \mathrm{MeV}\right)$ and $\left(\hbar \lambda_{p}=2 \mathrm{MeV}, \lambda_{q}=0\right)$ are presented by solid, dotted and dashed lines, respectively.

FIG. 2. Calculated dependence of the tunneling probability $P$ on the friction coefficient in momentum $\lambda_{p}$ at temperatures $k T=0$ and $3 \mathrm{MeV}, \hbar \omega=2.0 \mathrm{MeV}, q(0)=-2 \mathrm{fm}$, $\sigma_{q q}(0)=0.2 \mathrm{fm}^{2}, E=-5 \mathrm{MeV}, m=53 m_{0}$, and friction coeffisients in coordinate $\lambda_{q}=0$ $\left(\lambda=\lambda_{p} / 2\right)$. The calculations for the cases $\left(k T=0, D_{q q}=\hbar \lambda /(2 m \omega), D_{p p}=\lambda m \hbar \omega / 2\right.$ and $\left.D_{p q}=0\right),\left(k T=0, D_{q q}=0, D_{p p}=\lambda_{p} m \hbar \omega / 2\right.$ and $\left.D_{p q}=0\right),(k T=3 \mathrm{MeV}$, $D_{q q}=\hbar \lambda /(2 m \omega) \operatorname{coth}(\hbar \omega /(2 k T)), D_{p p}=\lambda m \hbar \omega / 2 \operatorname{coth}(\hbar \omega /(2 k T))$ and $\left.D_{p q}=0\right)$ and $(k T=3$ $\mathrm{MeV}, D_{q q}=0, D_{p p}=\lambda_{p} m \hbar \omega / 2 \operatorname{coth}(\hbar \omega /(2 k T))$ and $\left.D_{p q}=0\right)$ are presented by solid, dashed, dotted and dashed-dotted lines, respectively.

FIG. 3. Calculated dependence of the penetration probability $P$ on the initial variance $\sigma_{q q}(0)$ at $k T=0, \hbar \omega=2.0 \mathrm{MeV}, q(0)=-1 \mathrm{fm}, p(0)=0, m=53 m_{0}, D_{q q}=\hbar \lambda /(2 m \omega), D_{p p}=\lambda m \hbar \omega / 2$ and $D_{p q}=0$. The results obtained with $\lambda=\lambda_{p}=\lambda_{q}=0$ and $\hbar \lambda=\hbar \lambda_{p}=\hbar \lambda_{q}=1 \mathrm{MeV}$ are presented by solid and dashed lines, respectively. The value $\sigma_{q q}(0)=\hbar /(2 m \omega)$ is marked by arrow.

FIG. 4. Calculated dependence of the decay time from the metastable state in the potential (11) on the friction coefficient $\lambda_{p}$ at $\lambda_{q}=0\left(\lambda=\lambda_{p} / 2\right), k T=0, p(0)=0, \sigma_{q q}(0)=0.2 \mathrm{fm}^{2}$ and $m=53 m_{0}$. The depth of potential pocket with the minimum at $q(0)=-1.08 \mathrm{fm}$ is $5 \mathrm{MeV}$ $\left(\alpha=-2.57 \mathrm{MeV} \mathrm{fm}^{-2}\right.$ and $\left.\beta=1.59 \mathrm{MeV} \mathrm{fm}^{-3}\right)$. The top of the barrier corresponds $E=0 \mathrm{MeV}$ at $q=0 \mathrm{fm}$. The calculations for the cases $\left(D_{q q}=\hbar \lambda /(2 m \omega), D_{p p}=\lambda m \hbar \omega / 2\right.$ and $\left.D_{p q}=0\right)$ and $\left(D_{q q}=0, D_{p p}=\lambda_{p} m \hbar \omega / 2\right.$ and $\left.D_{p q}=0\right)$ are presented by solid and dotted lines, respectively. 


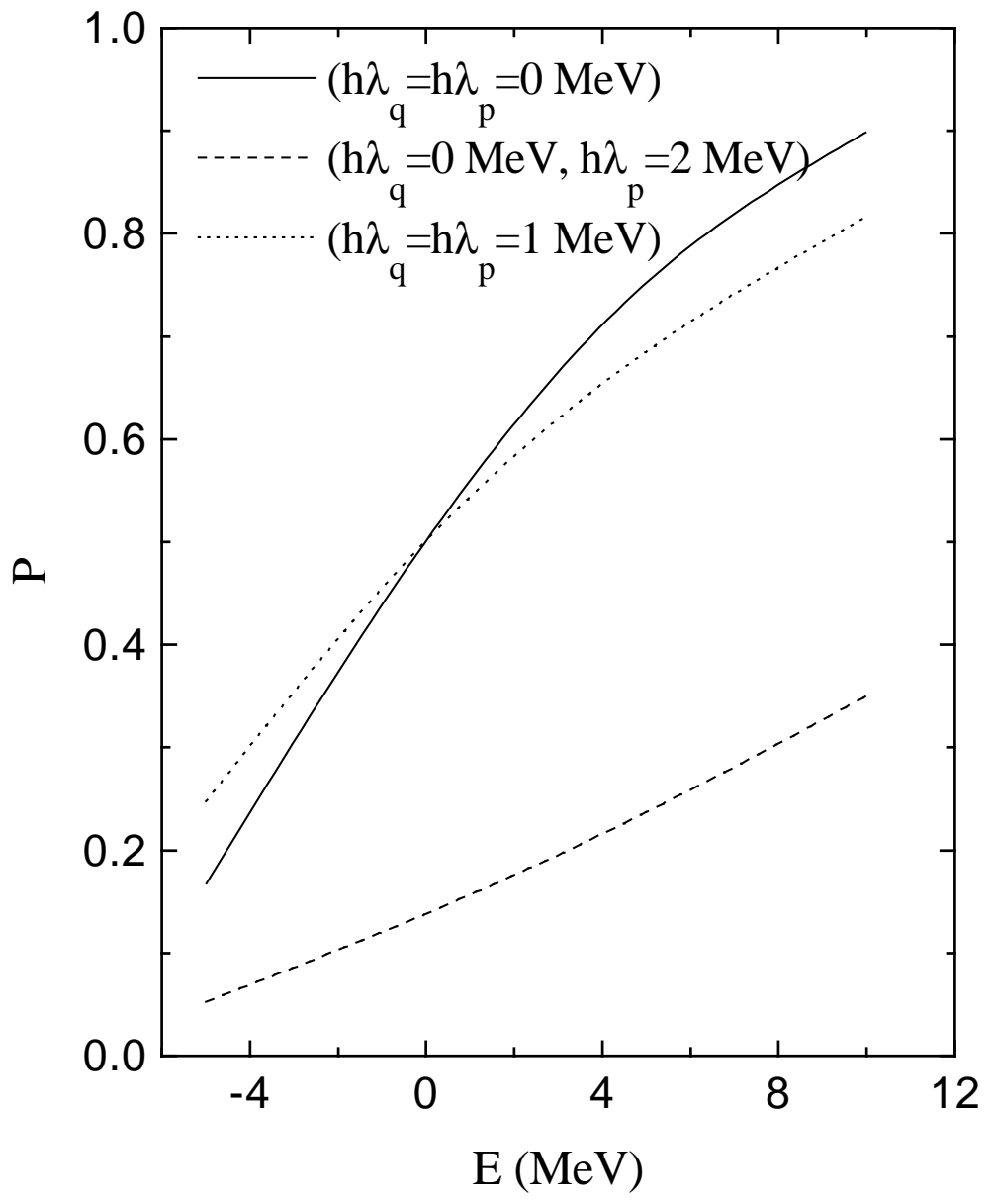




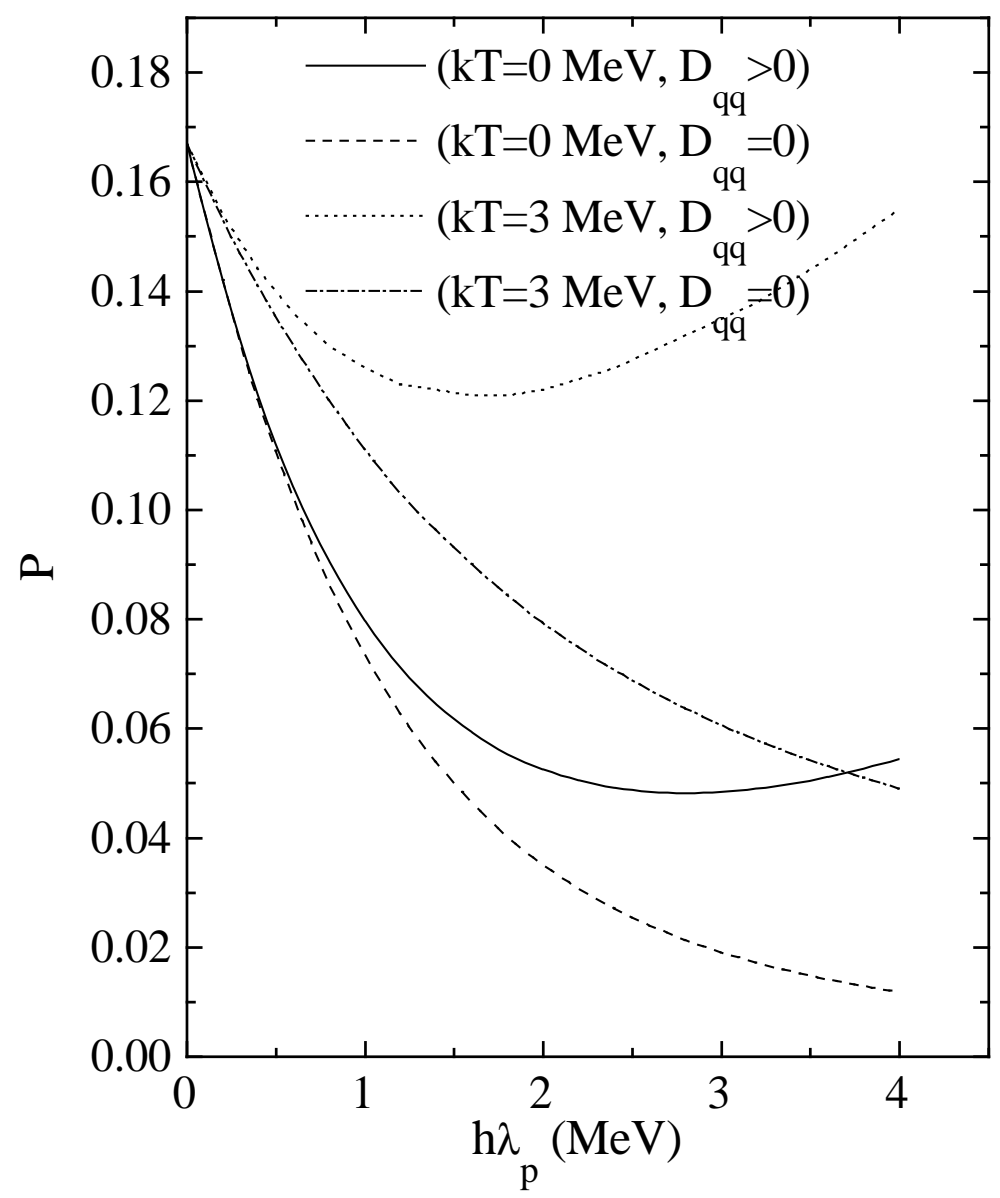




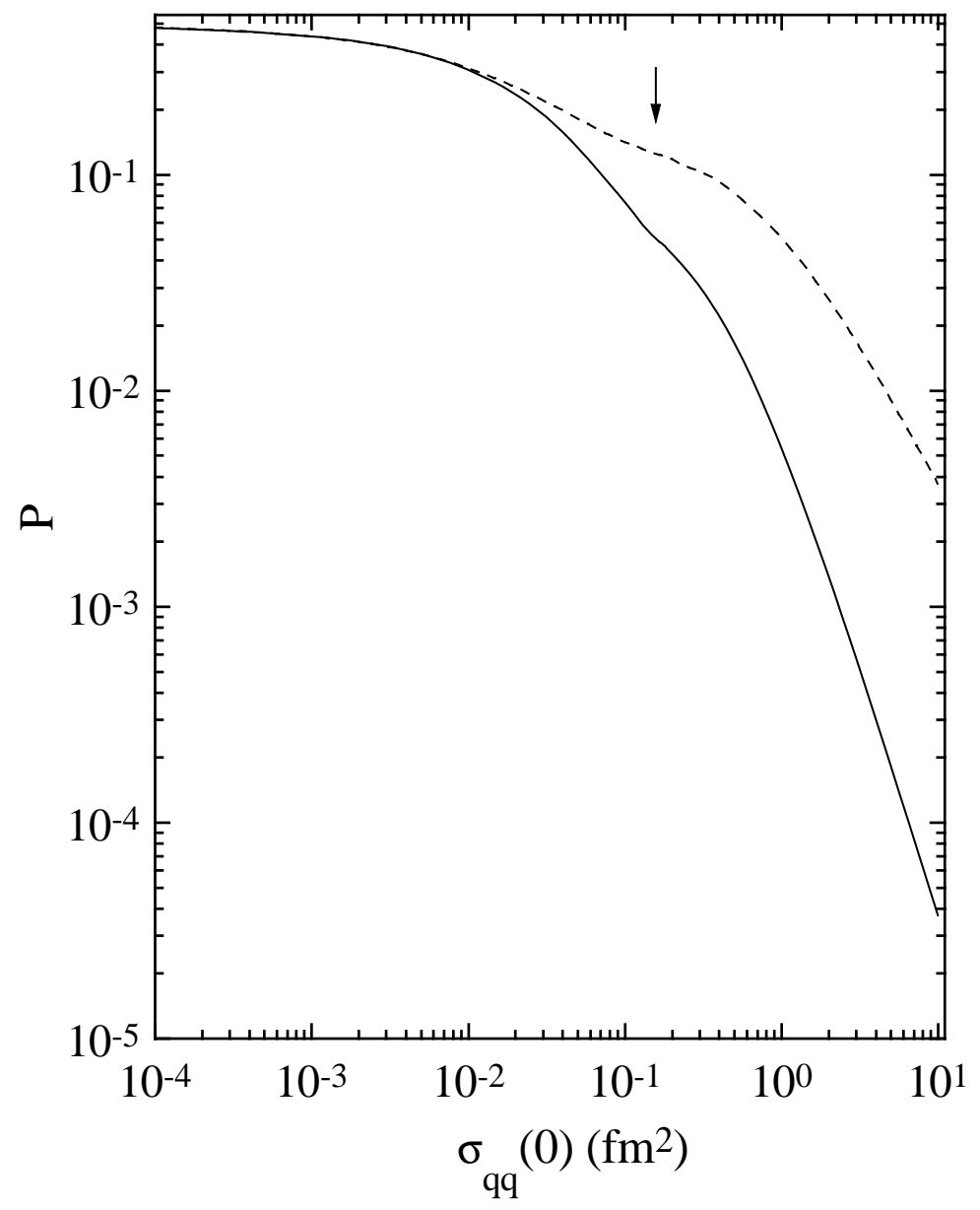




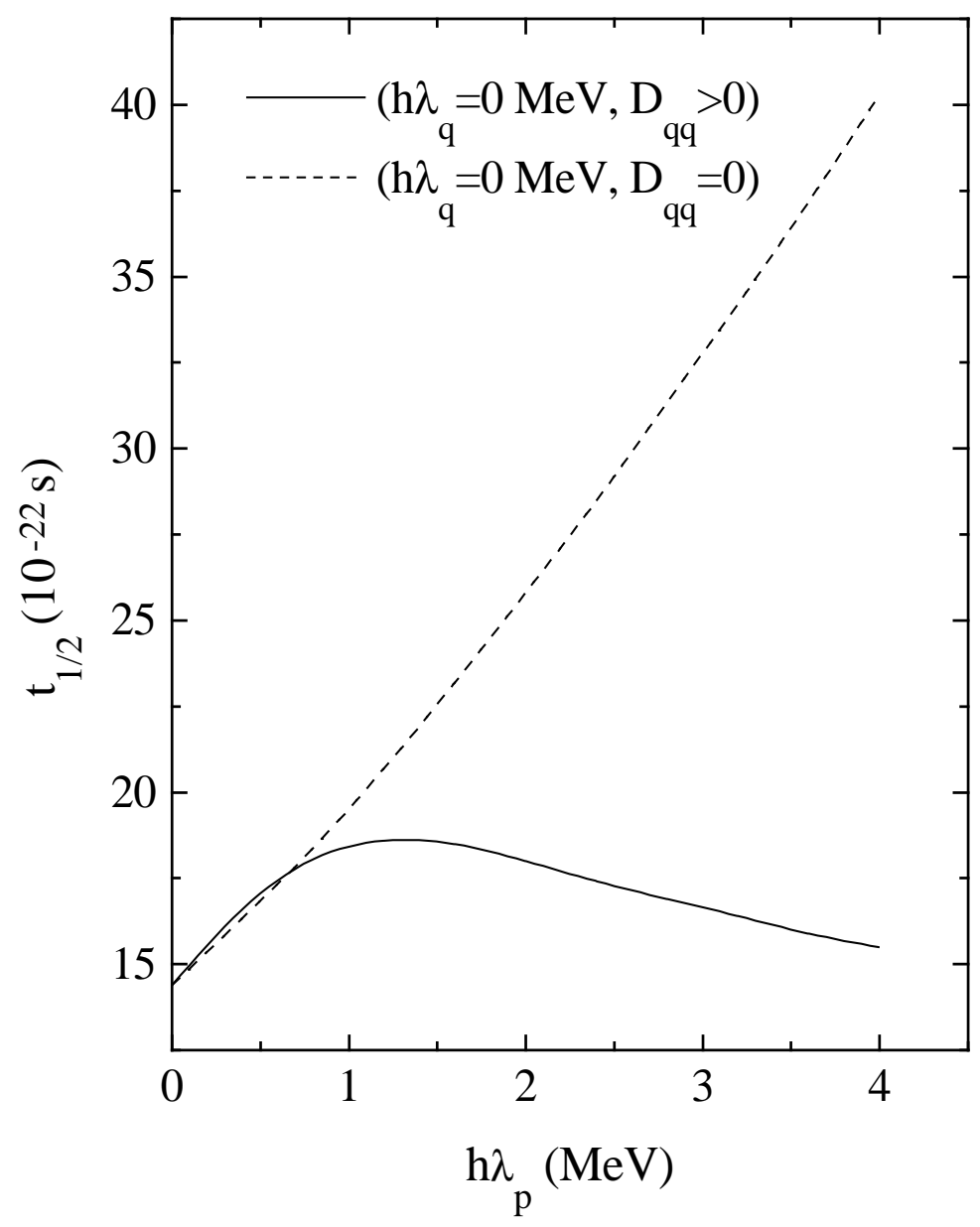

